\title{
The Application Value of Prenatal Ultrasound in the Diagnosis of Fetal Malformation
}

\author{
Xiangyi Xu, Chaohu Li \\ School of Medical Imaging, Bengbu Medical College, Bengbu 233030, Anhui, China \\ Email: 1130728558@qq.com
}

\begin{abstract}
Ultrasound examination during pregnancy has become an important part of prenatal care in China and even the world, and it has a vital diagnostic role for malformations and lesions of various fetal systems. A variety of fetal malformations can be treated in utero after prenatal diagnosis or timely treatment after delivery. However, many congenital diseases cannot be confirmed by ultrasound diagnosis, or the prenatal ultrasound performance is inconsistent with the postpartum clinical outcome, which has become a shortcoming of ultrasound diagnosis during pregnancy. This article reviews the main points of ultrasound diagnosis of common fetal malformations.
\end{abstract}

Keywords: prenatal ultrasound, deformity, diagnosis

\section{Introduction}

Fetal malformation refers to a structural or chromosomal abnormality that occurs in the uterus, which can lead to the death or severe disability of the fetus or newborn. There are many predisposing factors for fetal malformations, and the main factors include external factors and genetic factors. Early diagnosis and detection of fetal malformations can effectively reduce the fetal mortality ${ }^{[1]}$. The causes of fetal malformations are complex and diverse, including fetal genetic factors, maternal or external environmental factors, etc. Prevention of fetal malformations, timely detection of serious fetal malformations and induction of labor are important means to improve the quality of the birth population.

\section{Ultrasound diagnosis of infant deformities}

\subsection{Ultrasound diagnosis of cardiac malformations}

The incidence of infant heart malformations accounts for about $0.4 \%$ to $1.0 \%$ of live births. It is caused by abnormal development of the fetus in the mother's body and is closely related to factors such as genetics, environment, viral infections, and amniotic diseases. Heart malformations can threaten the normal growth and development of the fetus. In severe cases, lung infection and heart failure may occur, increasing the risk of fetal death ${ }^{[2]}$. Therefore, making a clear diagnosis of the condition in its prenatal examination and taking timely countermeasures is essential to reduce the incidence of heart disease ${ }^{[3]}$. At present, the main method for prenatal malformations of fetal cardiac ultrasound is the three-segment method of the heart. At 10 weeks of gestation, the fetus's heart has basically formed. Ultrasound can be used to observe the structure of the fetus's heart. Transvaginal ultrasound has better results. The examination includes left and right ventricular outflow tract views, tracheal views, four-chamber heart views, and three blood vessels. Common fetal heart malformations include single ventricle, endocardial pad defect, aortic stenosis, and tetralogy of Fallot. It also has characteristic ultrasound performance. For example, when the fetus with a single ventricle is 23 weeks gestational, echocardiography can find that the heart section is a single ventricle with no ventricular septal echo. The four-chamber view of the color Doppler ultrasound shows two beams of color. The blood flow into the single ventricle ${ }^{[4]}$. Endocardial cushion defect is a heart septal defect, that is, atrioventricular septal defect ${ }^{[5]}$. It is divided into complete endocardial cushion defect and partial endocardial cushion defect. Complete defect means that there is only one entrance between the atrium and the ventricle. The four-chamber view of the heart shows that the central fibrous body of the heart disappears, and the junction of the atrial septum and the interventricular septum The echo is interrupted, and a group of atrioventricular valves is moving in the heart cavity. The cross image formed by the atrioventricular septum and the atrioventricular valve disappears, and Doppler shows the blood flow between the four atrioventricular communication with each other. Valve regurgitation may also occur during systole. It should be distinguished from single ventricular malformations. Partial endocardial cushion defects have two entrances between the atria and the ventricles, and the left and right atria are connected to each other. There is echo loss in the lower part of the atrial septum on the four-chamber heart view, the attachment points of the mitral and tricuspid valves at the ventricular septum are 
at the same level, and the secondary and tricuspid valves have regurgitant signals. These indicate that ultrasound examination has important value in the examination of early fetal heart malformations.

\subsection{Ultrasound diagnosis of urinary system malformations}

Fetal urinary system malformations account for a relatively high proportion of congenital malformations in each system, about $15 \%$ to $20 \%{ }^{[6]}$. It is closely related to the survival and prognosis of the fetus. Ultrasound diagnosis has the advantages of simple, high-efficiency, non-invasive examination, etc. It can more intuitively show the growth and development of the fetus during pregnancy. By scanning the fetal kidney and bladder, the growth and development of the fetal urinary system can be judged, and it can be screened early before delivery. Check that early and correct treatment can alleviate patient suffering and reduce neonatal mortality ${ }^{[7,8]}$. The extensive use of ultrasound technology in clinical diagnosis is of great significance for effectively improving the detection rate of fetal urinary system malformations and achieving prenatal and postnatal care ${ }^{[9]}$. Common fetal urinary system malformations include hydronephrosis, renal failure, polycystic kidney disease and other diseases.

Some fetuses with hydronephrosis may have no obvious abnormal symptoms after birth. Ultrasound at 28 weeks of pregnancy can show that the anterior and posterior diameter of the renal pelvis is $10-13 \mathrm{~mm}$, the junction of the ureter and renal pelvis is narrow, the renal pelvis and renal calyces are all dilated, the ureter is not dilated, and the renal calyx is surrounded In the vicinity of the renal pelvis, the liquid dark area shows a "petal-like" renal parenchyma thinning ${ }^{[10]}$. Kidney failure includes the absence of both kidneys and the absence of unilateral kidneys. When both kidneys are absent, the kidney fossa, pelvis, abdomen and thoracic cavity can not show the ultrasound image of the fetal kidney. The adrenal glands are relatively enlarged, with a "supine" sign. Color Doppler ultrasound cannot show bilateral renal arteries. The fetal bladder cannot be displayed because it has not been filled for a long time. When unilateral kidney is absent, the kidney image on the absent side cannot be detected by ultrasound scan, and the kidney on the normal side becomes compensatoryly enlarged. Doppler ultrasound showed that the renal artery on the affected side was absent, while the renal artery on the contralateral side was present, the fetal bladder showed good, and the amniotic fluid volume was normal. The main ultrasound features of polycystic kidney disease are bilateral kidney enlargement, accompanied by substantial echo enhancement, and multiple vesicle structures of varying sizes can be seen in the kidney area. Polycystic kidney dysplasia often needs to be differentiated from hydronephrosis. Cystic lesions do not communicate with each other, and there is no functional intermediary substance between cysts. After childbirth, it is found that the cortex has lost normal function through nuclide nephrogram examination ${ }^{[11]}$. Multiple cystic dysplasia often

There are characteristic ultrasound findings. The scan shows that the ultrasound image on the side of the lesion, the multilocular cystic mass replaces the normal shape of the kidney. The cysts in the masses are of different sizes and shapes, and the cysts are not connected to each other., Between the capsules, there are often masses or small islands of substantial tissue. When the capsule enlarges, the kidney contour can become a grape cluster. There is no normal renal cortex around the kidney, and the collecting system has no normal echo. Doppler ultrasound shows that the branches of the renal artery in the kidney are disordered, and it is difficult to display the renal artery. The arterial spectrum is a high-impedance spectrum. Ultrasound is used as a routine examination method for fetal urinary system malformations in the second and third trimesters, which can detect some diseases in time before delivery and effectively improve the physical fitness of newborns.

\subsection{Deformities of the musculoskeletal system}

Fetal musculoskeletal system malformations and limb malformations are also common in clinical practice. A variety of reasons can cause limb deformities. There are many types of fetal limb deformities and various clinical manifestations. The toes and fingers of the fetus can be identified by ultrasound at 11 gestational weeks ${ }^{[12]}$. Therefore, early prenatal ultrasound detection can screen some skeletal abnormalities of the fetus limbs. In recent years, the accuracy of ultrasound detection of such abnormalities has been significantly improved. When ultrasound scans the fetus's whole body bone condition, the bone length should be measured, and the degree of bone mineralization and bone morphology should be observed. Fetal musculoskeletal system and limb malformations can be divided into generalized skeletal or cartilage dysplasia and localized skeletal malformations, and generalized bones are divided into fatal skeletal dysplasia (eg: fatal dwarf, osteogenesis imperfecta type II, limb flexion), Achondroplasia, non-fatal dysplasia (such as: achondroplasia, osteogenesis imperfecta type I, type III, type IV) ${ }^{[13]}$. Fatal skeletal dysplasia has common ultrasound manifestations, such as severely short and curved limbs, long bones of the limbs are 4 standard deviations below the average of the normal gestational week, the bust circumference is lower than the average of the normal gestational week, and the cardiothoracic ratio $>60 \%$, etc. There are also some special signs such as "earpiece-shaped" long bones and "clover" skull fractures. The long bones of the fatal dwarf children were significantly shortened, and the metaphysis was thick and looked like a "telephone handset". In addition, skin 
thickening and edema, serous effusion, and abnormal posture and movement of the fetus in the uterus may also occur; the long bones of the limbs of patients with achondroplasia are extremely short, the echo intensity is weakened, and the sound shadow behind the bones is not obvious. The thoracic cavity is narrow, the abdomen is bulging, and the vertebral body and the triangular ossification centers in the vertebral arches on both sides cannot be shown in the transverse section. The head is enlarged, and the double parietal diameter and head circumference are out of proportion to the gestational age. The fetus may have systemic edema, serous effusion, and polyhydramnios. Children with non-fatal bone dysplasia can be screened in prenatal ultrasound diagnosis, but it is difficult to accurately distinguish their specific types. The main ultrasound findings are: mild to moderate short limbs, some children with short limb symptoms only appear in the third trimester, may appear forehead bulge, horizontal ribs, narrow chest, non-progressive narrow chest may be accompanied by other deformities, such as multiple posterior axis Finger, lower jaw, foot varus, congenital heart disease, cleft lip (palate), etc. Ultrasound can also be used for prenatal diagnosis of limb deformities, such as ulnar and radius dysplasia or loss, fixed or abnormal hand posture, abnormal fingers, varus, abnormal toes, abnormal knee joint development, etc. Prenatal ultrasound can be used as the first choice for screening fetal limb deformities, with less damage, safety, convenience, and high diagnostic value. When the structure of the fetus is found to be abnormal, molecular genetics can be performed to find the cause.

\section{Limitations of prenatal ultrasound}

As an important imaging examination tool, ultrasound also has its limitations. Although most fetal malformations can be detected by prenatal ultrasound, the accuracy cannot reach 100\%. The detection rate of polydactyly (toe) deformities, small lips, cleft palate and other diseases is still low. In addition, many factors such as fetal position, normal amniotic fluid volume and the thickness of the mother's abdominal wall will affect the prenatal detection of fetal malformations ${ }^{[14]}$.

\section{Summary}

Ultrasound examination technology has been widely used in prenatal screening and diagnosis; the operation of sonographers has become more and more standardized; the detection rate of fetal abnormalities and the accuracy of diagnosis have been significantly improved. To a certain extent, this has avoided the birth of fetuses with severe congenital diseases, reduced birth defects, and improved the physical fitness of newborns in our country. At the same time, some problems have been exposed in the early prenatal ultrasound screening, which requires further improvement and innovation ${ }^{[15]}$.

\section{References}

[1] Zhao Yajian. The diagnostic value of prenatal ultrasound for fetal malformations[J]. South China Journal of Medicine, 2020,18(11):148-149.

[2] Yang Xinyan. Application of color Doppler ultrasound screening in prenatal diagnosis of fetal heart malformations[J]. Imaging Research and Medical Applications, 2020,4(21):181-182.

[3] Song Hualei, Fang Qun, Chen Baojiang, et al. Prenatal diagnosis and prognosis of fetuses with isolated mild lateral ventricle widening[J]. International Journal of Obstetrics and Gynecology, 2012,39(1):90-91.

[4] Wang Wanting, Zheng Ruilian, Hu Wenjiang, Wei Yun. Application value analysis of prenatal ultrasound in screening fetal congenital heart disease[J]. Imaging Research and Medical Applications, 2019,3(19):202-203.

[5] Fan Chunfang. Prenatal ultrasound diagnosis of fetal urinary system malformations[J]. Everyone's Health (Academic Edition), 2016,10(14):55.

[6] Wang Xiaoyu, Li Haiming. The application and clinical significance of fetal system ultrasound in the screening of prenatal fetal malformations[J]. China Maternal and Child Health Care, 2015,30(26):4517-4519.

[7] Jing Chunli, Li Jing, You Shuyan, et al. Retrospective analysis of misdiagnosis cases of prenatal ultrasound misdiagnosis of fetal urinary system malformations[J]. Chinese Journal of Ultrasound in Medicine, 2017,(4):329-331.

[8] He Huanrong. The diagnostic effect of ultrasound on fetal urinary tract malformations[J]. Chinese Journal of Family Planning, 2015,23(12):855-858.

[9] Wang Shuyun, Tong Yajun, Kang Hongxia, Yin Shuhong, Li Juan. The clinical application value of echocardiography in the diagnosis of fetal endocardial cushion defects[J]. China Medical Herald, 2012,9(03):168-169.

[10] Cao Min, Wang Jingsheng, Su Hong, Wang Junli, Huang Bo. Ultrasonic diagnosis and clinical value of fetal urinary system malformations in the second and third trimester of pregnancy[J]. Chinese Journal of Eugenics and Genetics, 2018,26(12):100-103.

[11] He Huanrong. The diagnostic effect of ultrasonography on fetal urinary tract malformations[J]. Chinese Journal of Family Planning, 2015,23(12):855-858. 
[12] Zhao Xia, Wang Ling, Wang Jinqiang, et al. The clinical value of prenatal ultrasound examination of the fetus at 11-14 weeks [J]. Harbin Medicine, 2017,37(3):259-260.

[13] Li Min, Tang Fengzhen, Yu Qinghong, Bai Yanhong. Prenatal ultrasound diagnosis method and evaluation of fetal limb malformations[J]. Journal of Aerospace Medicine,2012,23(04):453-455.

[14] Yan Xingyan, Huang Chaoning. Research progress of prenatal ultrasound diagnosis of fetal congenital heart disease[J]. Modern Medicine and Health, 2016,32(05):702-704.

[15] Su Jing. Research progress in early prenatal ultrasound screening and diagnosis[J]. China Medical Device Information, 2019,25(09):51-52. 